\title{
Guardian de las alturas andinas. Estado de conservación actual del condor andino ( Vultur Gryphus)
}

\author{
Guardian of the Andean heights. Conservation status of the Andean condor \\ (Vulture Gryphus)
}

Jorge Simón Tanquero

DOI. $10.21931 / \mathrm{RB} / 2016.01 .04 .10$

\section{RESUMEN}

Sobrevolando la cordillera de los Andes, el Cóndor Andino, Vultur Gryphus de la familia Cathartidae, se destaca como el ave no marina de mayor envergadura del planeta, especie endémica de la zona y emblema nacional de numerosos países suramericanos vinculado al folclore. Sus benignos hábitos carroñeros mantienen limpias largas extensiones montañosas, páramos y desiertos, evitando la proliferación de enfermedades, sin embargo, en la actualidad la falta de una adecuada difusión de información sobre esta especie y la agresiva invasión de su hábitat causan que su conservación se encuentre en estado crítico, siendo la presencia humana el principal factor de mortalidad. Por ello, desde hace varias décadas, a través de la labor conjunta internacional se ha avanzado significativamente en el estudio, conservación y repoblamiento de antiguos hábitats, evitándose la pérdida irreversible de las poblaciones, considerándose la limitada capacidad de respuesta rápida demográfica de esta ave. Para ello, una continua y activa divulgación de la realidad y la lucha del Cóndor Andino han ayudado a evitar que sus características biológicas en conjunto a las amenazas humanas que enfrentan se conviertan en una combinación irremediable para su supervivencia.

Palabras clave: Vultur gryphus, Suramérica, conservación, vulnerabilidad.

\section{ABSTRACT}

Overflying the Andes Mountains, the Andean Condor, Vultur Gryphus of the Cathartidae family, stands out as the largest non-marine bird on the planet, an endemic species of the area and national emblem of many South American countries linked through folklore. Their benign carrion habits keep clean long mountain ranges, moors and deserts, preventing the proliferation of diseases, however, at the present the lack of an adequate diffusion of information on this species and the aggressive invasion of its habitat cause that its conservation is found in critical condition, being the human presence the main factor of mortality. For this reason, for several decades, through joint international efforts, significant progress has been made in the study, conservation and repopulation of ancient habitats, avoiding the irreversible loss of populations, considering the limited demographic fast response capacity of this bird. To this end, a continuous and active dissemination of the reality and the struggle of the Andean Condor have helped to avoid that their biological characteristics, together with the human threats they face, become an hopeless combination for their survival.

Keywords: Vultur gryphus, South America, conservation, vulnerability.

\section{Introducción}

Como venerados dueños de las alturas, el Cóndor de los Andes (Vultur gryphus) se alza ante el majestuoso paisaje y el folclor andino como fuente de inspiración. Alcanzando fácilmente los 6 mil metros de altura, el Cóndor Andino se distingue en la distancia por su brillante plumaje negro-azulado y su gran envergadura. De cerca, la blanca gargantilla y una cabeza rosa desprovista de plumas son los rasgos que definen al cóndor, que en caso de ser macho adorna su cabeza con una cresta roja, carnosa y prominente. Llegando hasta los 3.2 metros de envergadura, los 130 centímetros de largo y pesando de 8 a 15 kilos no es de extrañar que por tal imponente presencia y los mitos que le acompañan, se le otorgara valor religioso en el pasado, apareciendo en innumerables cerámicas o pinturas rupestres. ${ }^{1}$ Valor que en el presente se manifiesta a través de su figura como símbolo nacional para varios países andinos, entre ellos Chile, Argentina y Ecuador.

Presente en las cordilleras de los Andes de América del Sur hasta las montañas Santa Martha, el cóndor se extiende desde Argentina hasta Venezuela, habitando acantilados, cuevas en altas montañas, pastizales, páramos e incluso desiertos, viajando hasta 200 kilómetros durante el día en busca de carroña y criaturas pequeñas o moribundas de la cuales se alimenta, labor para la cual se sirve de recias patas, afiladas garras y un aguzado sentido del olfato. Para detectar su comida, el cóndor se vale del olor de gases tales como el mer- 


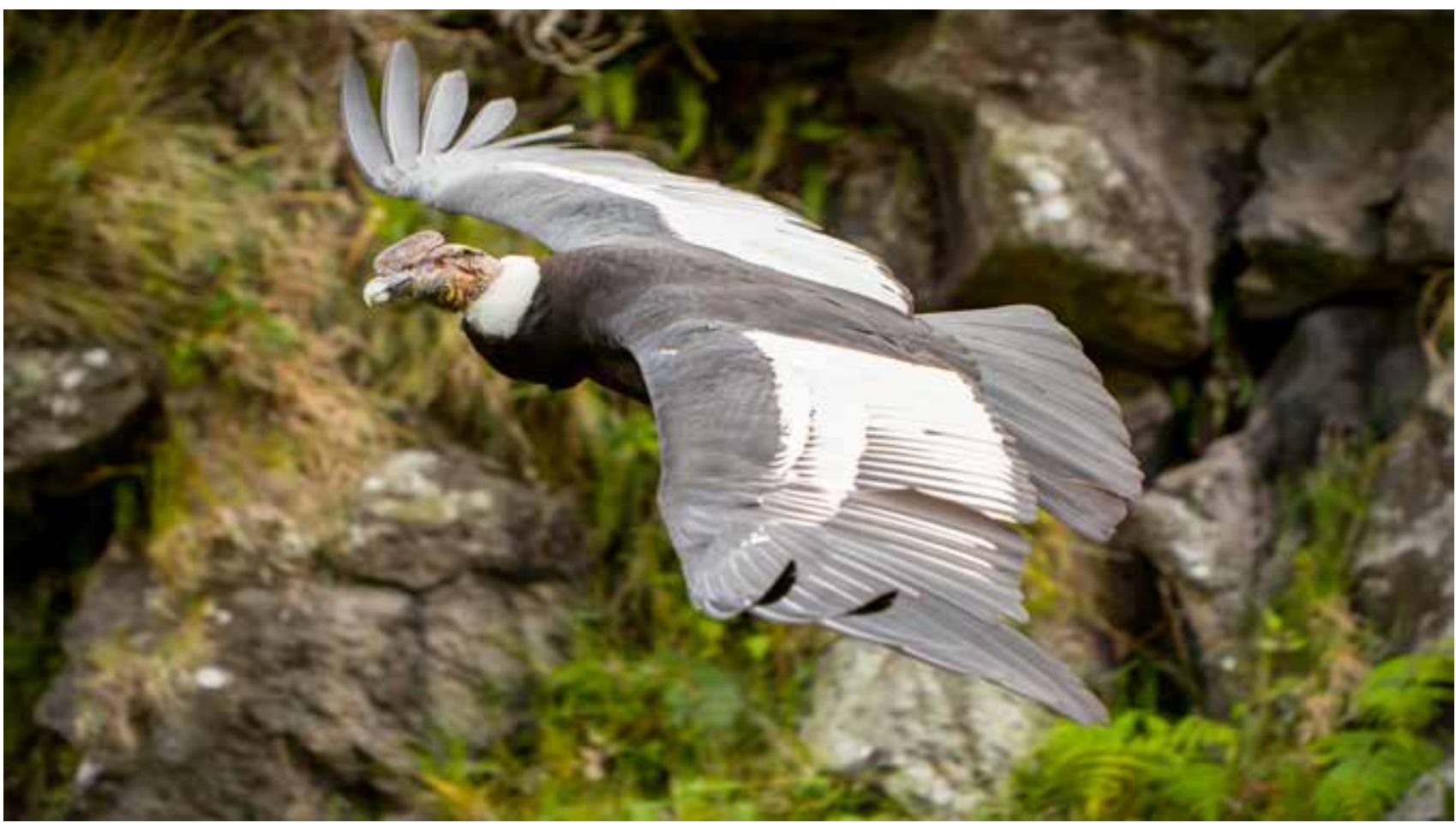

Cóndor de los Andes (Vultur gryphus)@ Ammit | Dreamstime.com Male Andean Condor In Flight Photo

captano de etilo que se desprende de cuerpos en descomposición, y para ello basa su dieta predilecta en mamíferos perecidos de gran tamaño como caballos, cabras, llamas y venados, los cuales no consume de inmediato, sino que puede esperar hasta tres días antes animarse a hacerlo. ${ }^{1}$ Son precisamente estas costumbres alimenticias las que mantienen al páramo y los valles libres de animales en putrefacción, evitan la proliferación de enfermedades, y dan nombre a la familia Cathartidae a la que pertenece el Cóndor, nombre que viene del griego kathartes que significa "el que limpia".

En el aspecto reproductivo, durante su vida, el Cóndor Andino es monógamo y conserva una única pareja. Curiosamente, en ocasiones se puede observar al macho durante el cortejo obsequiar a la hembra pequeños trozos de ramitas y hojas que luego colocan bajo sus alas, un peculiar comportamiento que solo se ha podido registrar en aquellos que viven en cautiverio. ${ }^{1}$ Una vez seleccionada la pareja, el macho puede vivir en el nido con la hembra hasta 2 meses antes de aparearse y 2 meses después de realizada la cópula, tiempo durante el cual ambos padres se turnan en el proceso de incubación de un único huevo. Una vez nacida la cría ambos padres proveen los cuidados necesarios hasta que el polluelo crece al tamaño de sus padres en el trascurso de 2 años. El joven cóndor por su parte no será sexualmente maduro hasta pasados los 8 años, llegando a tener propiamente solo una cría cada 2-3 años. ${ }^{3}$

En caso que los padres de una cría hayan fallecido o desaparecido, para mantener al polluelo vivo, se minimiza el contacto humano con la cría; alimentándose los polluelos con títeres de látex semejantes a cóndores adultos con el fin de evitar la impronta de los polluelos con los seres humanos, lo cual podría ponerlos en peligro al ser liberados, pues no reconocerían a los seres humanos como un peligro. Ya crecidos, los cóndores se mantienen en aviarios durante tres meses antes de su liberación en donde se aclimatan a un ambiente similar al entorno donde serán liberados. Una vez libres, se les da seguimiento por satélite con el fin de observar sus movimientos y cerciorarse que permanecen vivos. ${ }^{4}$

En la actualidad, la situación el Cóndor Andino en el Ecuador es crítica, "Casi Amenazado" según la Unión Internacional para la Conservación de la Naturaleza (UICN, 2013), pues se estima en la actualidad existen menos de 58 ejemplares en estado silvestre en el país. ${ }^{3}$ A pesar de su marcada longevidad, llegando a vivir hasta los 70 años, el cóndor se enfrenta a muy baja tasa de natalidad. A su vez, a pesar de no poseer depredadores naturales, la principal amenaza que enfrenta en toda su área de distribución es el hombre, a través de la caza furtiva incentivada por falsas creencias de lugareños sobre su supuesta actividad depredadora sobre el ganado, la destrucción progresiva de su hábitat mediante la expansión agrícola hacia los bosques alto andinos, el envenenamiento por la ingesta de carroña con cebos tóxicos o pesticidas y el agotamiento excesivo de especies de las cuales se alimenta ya sea por la caza indiscriminada o la presencia de especies introducidas, como lo son los perros domésticos o salvajes, que compiten por la carroña. ${ }^{5}$

En su estado silvestre el estado sanitario de las poblaciones es muy escaso, solo teniéndose algunos registros esporádicos que sugieren que en ciertos casos pueden sufrir de aspergilosis o sea, infecciones respiratorias de hongos pertenecientes al género Aspergillus. $^{5}$

Para su conservación en el Ecuador y restaurar a estas aves a su hábitat natural, se ha habilitado la labor conjunta de zoológicos y centros de rescate y conservación, además de fundarse el Grupo Nacional de Conservación del Cóndor, un censo de aves silvestres y cautivas enfocado en documentar el manejo de cóndores en cautiverio. ${ }^{6}$ Por su parte, el Quito Zoo (Quito) lidera un plan para compartir experiencias y obtener más pichones de cóndor en colaboración con el Proyecto Cóndor Huasi (Zuleta), el Parque Cóndor (Imbabura), el Zoológico San Martín de Baños (Tungurahua) y el Centro de Rescate Hacienda El Ilitío (Cotopaxi). Además de que se proyecta para un futuro un espacio ambientado en Zulueta para que jóvenes cóndores sean liberados con seguridad, contando con el apoyo de las comunidades que habitan los páramos. ${ }^{6}$

Aumentar el número existente de cóndores en el Ecuador se ha convertido en una labor más prometedora gracias a la Estrategia Nacional de Conservación del Cóndor Andino bajo programas que contemplan la capacitación de guardabosques en 


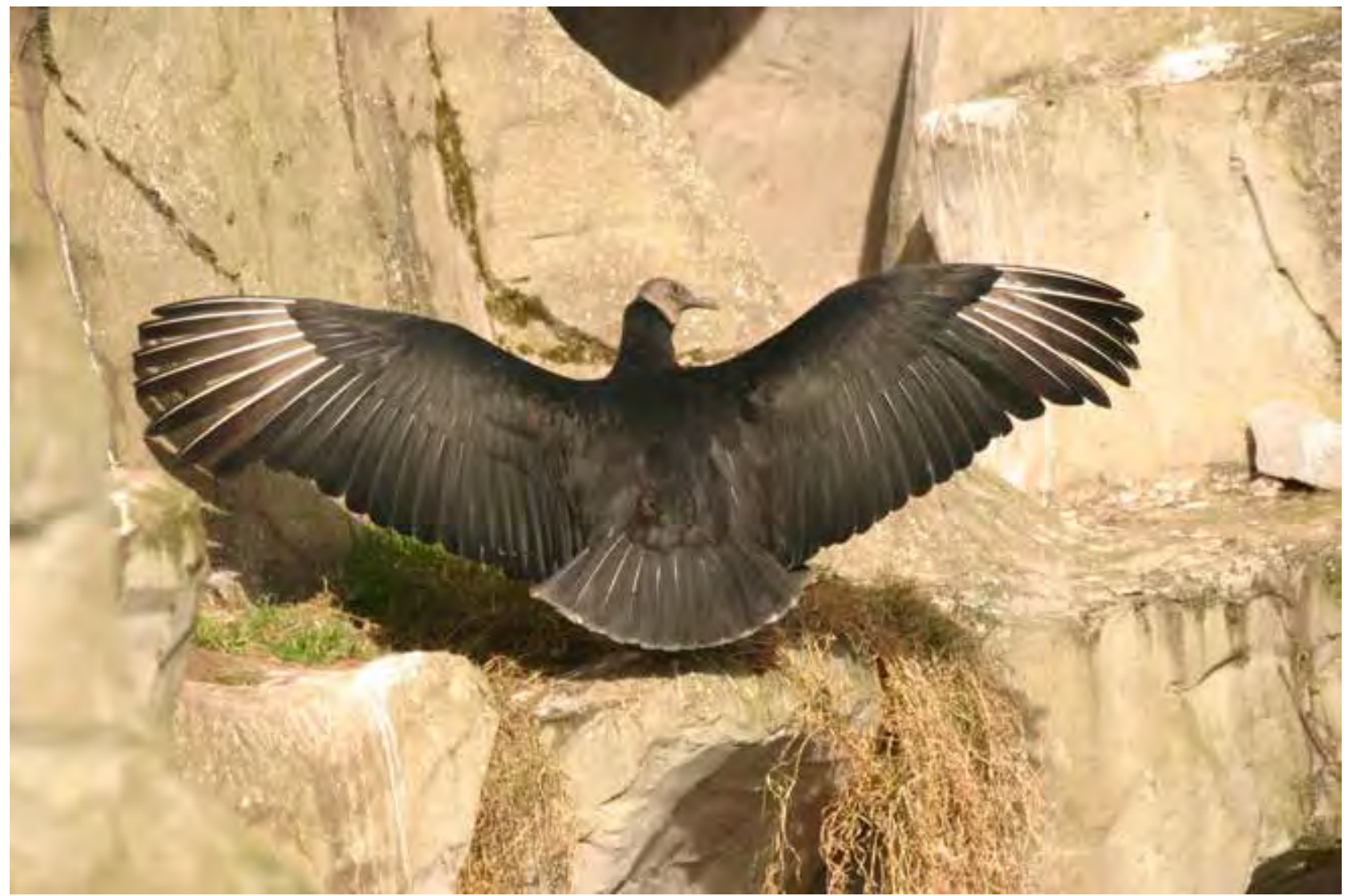

Andean Condor (Vultru gryphus) @ Ammit | Dreamstime.com perched on rocks. Photo taken on: March 09th, 2008

rescate y muestreo de individuos; la implementación de primeros auxilios para cóndores silvestres rescatados; y la reproducción y reintroducción de especímenes, además del apoyo del público con campañas de educación en el país que disuaden su cacería o captura.?

Por su parte, desde 1991 el zoológico de Buenos Aires y la Fundación Bioandina Argentina vienen trabajando en el Proyecto de Conservación Cóndor Andino, al cual se suman Aves Chile y el Parque Metropolitano de Santiago en el 2000 para crear el Programa Binacional de Conservación Cóndor Andino Chile-Argentina, donde se habilitó un centro de incubación artificial y cría en aislamiento humano para la posterior reintroducción de las aves. ${ }^{8}$ Recientemente este programa binacional también recolecta información sobre factores de amenaza para la especie a través de la evaluación física o necropsia de los cóndores hallados. ${ }^{5}$

La reincorporación de cóndores ha sido una estrategia utilizada por varios países desde mediados del 1990, siendo Argentina y Chile los países con mayor desarrollo de esta estrategia, lográndose liberar exitosamente individuos rehabilitados, así como aquellos criados en cautiverio, los cuales se han mantenido rastreados de forma satelital. ${ }^{8}$

Para Sur América en general, el Cóndor Andino representa un fragmento de su herencia cultural y un símbolo de su pasado y emblema de la civilización andina. Por ello su cuidado y preservación se ha vuelto ecológicamente indispensable, por su valor en la cadena trófica y por su magnífica estampa que se ha convertido en un ícono de fuerza, dedicación, orgullo y libertad con la que se identifica el pueblo latinoamericano.

Recibido: 20 de octubre de 2016.

Aprobado: 10 de diciembre de 2016.

\section{References}

1. http://www.bioenciclopedia.com/condor-de-los-andes/

2. Héctor F. Aguilar. "El cóndor de los andes Vultur griphus linnaeus, 1758 (ciconiiformes: ciconiidae: cathartinae): un visitante ocasional en Venezuela". Centro de investigación y reproducción de especies silvestres, Cires. Venezuela. Revista Ecol. Lat. Am. Vol. 6 No 3. 2000.

3. Pedro A. Ospina Salinas. "Situación del cóndor andino (Vultur gryphus) en Latinoamérica”. Universidad Nacional Mayor de San Marcos. Facultad de Medicina Veterinaria, Perú. 2013.

4. http://www.bioandina.org.ar/pcca Luis Jácome, Dir. Proyecto de Conservación Cóndor. Proyecto de Conservación Cóndor. 2016.

5. Sergio A. Lambertucci. "Biología y conservación del cóndor andino (Vultur gryphus) en Argentina". Laboratorio Ecotono, Universidad Nacional del Comahue. Argentina. 2007

6. http://www.quitozoo.org/index.php/fze/conservacion/condor-andino

7. Estrategia nacional para conservación del Cóndor Andino. Marcela Aguinaga Vallejo, Ministra del ambiente. Acuerdo Ministerial 51. 24-Julio-2009. Vigente. República del Ecuador.

8. Jácome L, Astore V. 2009. "Cuidados y desarrollo del primer pichón de cóndor andino nacido en la costa Atlántica patagónica “. Newsletter Conservación \& Ciencia No5, 01/11/2009. Jardín Zoológico de la Ciudad de Buenos Aires. Argentina 


\section{ESCUELA DE CIENCIAS BIOLÓGICAS E INGENIERÍA}

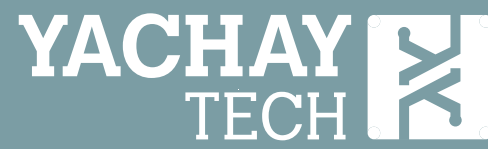

Yachay Tech es la primera universidad de investigación interdisciplinaria en Ecuador. Su objetivo es convertir a la región Andina en un centro que forme ciudadanos que contribuyan a la tecnología, la economía, la cultura y el espíritu empresarial del mundo.

La Universidad está en búsqueda de invesigadores Ph.Ds que deseen unirse al cuerpo docente de su Escuela de Ciencias Biológicas e Ingeniería y que deseen aportar activamente al desarrollo de la institución.

La escuela está liderada por el decano Spiros Agathos, PhD., cuenta con su propio Jardín Botánico para investigación, laboratorios de última tecnología y un importante equipo de trabajo que permite ampliar las conexiones de colaboración para la implementación de grandes proyectos.

Contacto de aplicaciones:

Spiros Agathos, Ph.D

sagathos@yachaytech.edu.ec
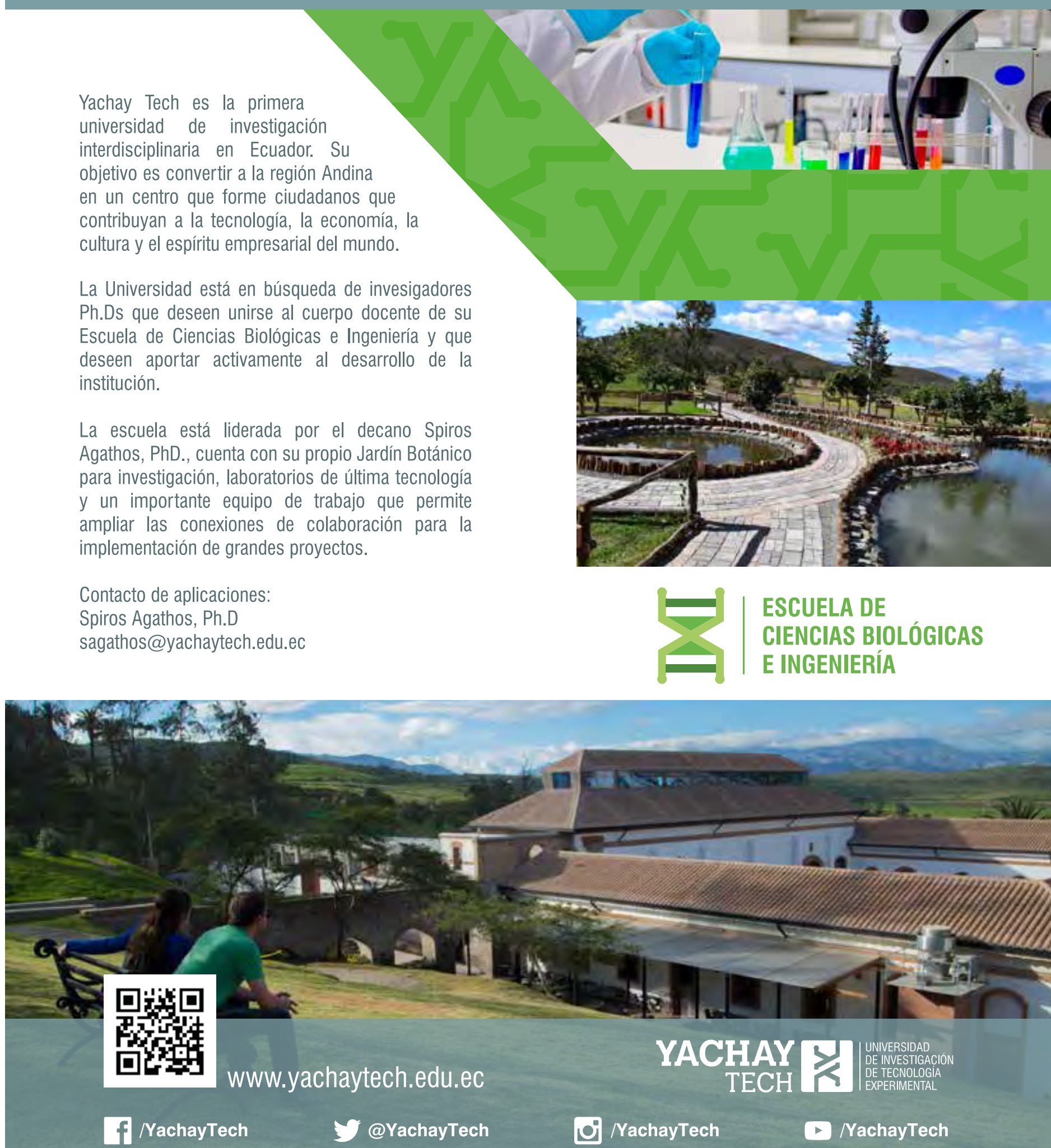